\title{
Formal solution to the KP hierarchy
}

\author{
S.M. Natanzon* $\quad$ A.V. Zabrodin ${ }^{\dagger}$
}

September 2015

\begin{abstract}
We find all formal solutions to the $\hbar$-dependent KP hierarchy. They are characterized by certain Cauchy-like data. The solutions are found in the form of formal series for the tau-function of the hierarchy and for its logarithm (the $F$-function). An explicit combinatorial description of the coefficients of the series is provided.
\end{abstract}

\section{Contents}

1 Introduction $\quad[2$

1.1 Motivation ........................... 2

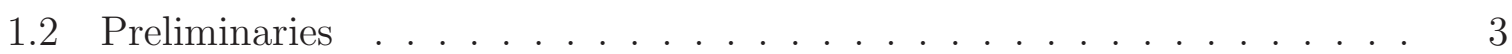

2 The $\hbar-K P$ hierarchy for the tau-function 6

2.1 Hirota equations for the tau-function . . . . . . . . . . . . 6

2.2 Formal solution for the tau-function . . . . . . . . . . . . . . . 7

3 The $\hbar$-KP hierarchy for the $F$-function $\quad 10$

3.1 Hirota equations for the $F$-function . . . . . . . . . . . . . . 10

3.2 The $\hbar$-KP hierarchy in an unfolded form . . . . . . . . . . . 11

3.3 The " $\hbar$-deformed partial derivatives" $\partial_{i}^{\hbar} \ldots \ldots \ldots \ldots$. . . . . . . . 12

3.4 The $\hbar$-KP hierarchy in terms of $\partial_{i}^{\hbar} \ldots \ldots \ldots$

*National Research University Higher School of Economics, 20 Myasnitskaya Ulitsa, Moscow 101000, Russia and ITEP 25 B.Cheremushkinskaya, Moscow 117218, Russia, e-mail: natanzons@mail.ru

†Institute of Biochemical Physics, 4 Kosygina st., Moscow 119334, Russia; ITEP, 25 B.Cheremushkinskaya, Moscow 117218, Russia and Laboratory of Mathematical Physics, National Research University Higher School of Economics, 20 Myasnitskaya Ulitsa, Moscow 101000, Russia, e-mail: zabrodin@itep.ru 
4.1 The Cauchy-like data . . . . . . . . . . . . . . . . . 15

4.2 Dual bases $h_{\lambda}$ and $m_{\lambda}$ and the formal solution $\ldots \ldots \ldots$

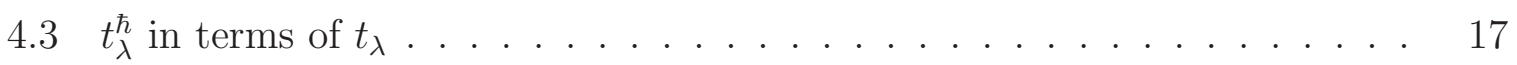

4.4 Algebra of $\hbar$-differential operators . . . . . . . . . . 18

4.5 Construction of formal solutions . . . . . . . . . . . . . 19

Appendix A: Proof of Proposition $2.2 \quad 20$

References

\section{Introduction}

\subsection{Motivation}

The partial differential equation (PDE)

$$
\frac{3}{4} u_{y y}=\frac{\partial}{\partial x}\left(u_{t}-\frac{3}{2} u u_{x}-\frac{1}{4} u_{x x x}\right)
$$

was derived by Kadomtsev and Petviashvili (KP) in 1970 for description of non-linear waves in two-dimensional media with small dispersion. Later it was recognized that this equation is integrable and it can be naturally embedded into an infinite system of compatible PDE's. This system is now called the KP integrable hierarchy. The KP hierarchy can be most naturally formulated in terms of bilinear equations for the taufunction $\tau=\tau\left(t_{1}, t_{2}, t_{3}, t_{4}, \ldots\right)$ of the infinite set of "times" $\mathbf{t}=\left\{t_{1}, t_{2}, t_{3}, \ldots\right\}$, the first three of which are identified with $x, y, t$ as $t_{1}=x, t_{2}=y, t_{3}=t$. The variable $u$ in equation (11) is expressed through the tau-function as $u=2 \partial_{x}^{2} F$, where $F=\log \tau$.

The KP hierarchy has a lot of exact solutions of very different nature: non-linear waves and excitations in dispersive media (quasi-periodic and soliton solutions [6, 20]), generating functions for topological invariants in algebraic geometry [27, 9], partition functions for models of random matrices [14], etc.

The tau-function admits the well-known expansion in Schur functions depending on the times $t_{i}$ [21. The structure of this expansion is studied in detail and well understood [2, 5, 4. However, in many cases it is the $F$-function which is of prime interest rather than the tau-function itself. At the same time, series expansions for the $F$-function are missing in the literature. This is the problem that we address in the present paper. It should be noted that we deal with not necessarily convergent series. The corresponding solutions are called formal.

One can introduce an auxiliary formal parameter $\hbar$ by re-scaling $t_{k} \rightarrow t_{k} / \hbar$, then the $\mathrm{KP}$ equation acquires the form

$$
\frac{3}{4} u_{y y}=\frac{\partial}{\partial x}\left(u_{t}-\frac{3}{2} u u_{x}-\frac{\hbar^{2}}{4} u_{x x x}\right)
$$


The corresponding re-scaling in the whole hierarchy is called the $\hbar$-formulation of the KP hierarchy (or the $\hbar$-dependent KP hierarchy [22]). The coefficients of its formal solutions are formal series in $\hbar$. The $F$-function is defined as $F=\hbar^{2} \log \tau$.

The parameter $\hbar$ has a meaning of the Planck's constant in the "auxiliary space", i.e., the space where the Lax operator of the KP hierarchy acts. The limit $\hbar \rightarrow 0$ is the quasiclassical limit in the auxiliary space. In the "physical space" of dynamical variables this limit is realized as the dispersionless limit, where higher space-time derivatives of dynamical variables are set to be negligibly small. For example, equation (2) at $\hbar=0$ becomes the Khokhlov-Zabolotskaya equation (the dispersionless KP equation).

The $\hbar \rightarrow 0$ limit of the KP hierarchy is called the dispersionless KP (dKP) hierarchy. It is a particular case of general Whitham hierarchy of PDE's [7, 8]. The dispersionless limit is of great interest on its own. (For applications to interface dynamics and problems of complex analysis see [12, 13, 28, 29, 19] and [26, 30] respectively.) A class of solutions to the dKP hierarchy can be obtained as the $\hbar \rightarrow 0$ limits of solutions to the $\hbar$-dependent KP hierarchy. However, such limits do not always exist in the class of smooth functions.

In the present paper we construct all formal solutions to the $\hbar$-dependent KP hierarchy in the form of an infinite formal series with the coefficients defined by an explicit recurrence procedure. The solution is determined by an infinite set of arbitrary functions of one variable $f_{0}(x), f_{1}(x), f_{2}(x), \ldots$ which are supposed to be formal series or differentiable infinitely many times. These functions are initial data for the solution. In particular, $F(x ; \mathbf{0})=f_{0}(x)$. However, the functions $f_{i}$ with $i \geq 1$ coincide with the

standard Cauchy data $\left.\partial_{t_{i}} F(x, \mathbf{t})\right|_{\mathbf{t}=0}$ only at $\hbar=0$. At $\hbar \neq 0$ these functions differ from the first order derivatives by some terms which are of higher order in $\hbar$. We call the set $\left\{f_{0}(x), f_{1}(x), f_{2}(x), \ldots\right\}$ the Cauchy-like data.

The fact that solutions to the KP hierarchy can be restored from the first order derivatives of the $F$-function at $\mathbf{t}=0$ was pointed out earlier [3, 17, 18]. However, no explicit solution of the Cauchy problem for the KP hierarchy is available even in the sense of formal series. In this paper we give explicit formulas that allow one to restore the formal solution from the arbitrary set of the Cauchy-like data $\left\{f_{0}(x), f_{1}(x), f_{2}(x), \ldots\right\}$.

\subsection{Preliminaries}

Here we list the necessary notations and facts related to Young diagrams and Schur functions.

Young diagrams. Following [10], we will denote the Young diagrams as $\lambda$, $\mu$, etc. Let $\lambda=\left[\lambda_{1}, \lambda_{2}, \ldots, \lambda_{\ell}\right]$ be the Young diagram with $\ell=\ell(\lambda)$ rows of non-zero lengths $\lambda_{1} \geq \lambda_{2} \geq \ldots \geq \lambda_{\ell}>0$. We identify $\lambda$ with the partition of the number $|\lambda|:=\lambda_{1}+\ldots+\lambda_{\ell}$ into the $\ell$ non-zero parts $\lambda_{i}$. Another convenient notation is $\lambda=\left(1^{m_{1}} 2^{m_{2}} \ldots r^{m_{r}} \ldots\right)$, which means that exactly $m_{i}$ parts of the partition $\lambda$ have length $i: m_{i}=\operatorname{card}\left\{j: \lambda_{j}=i\right\}$. In particular, the Young diagram with one row (respectively, one column) of length $k$ is denoted as $(k)$ (respectively, $\left.\left(1^{k}\right)\right)$. Put

$$
\rho(\lambda)=\lambda_{1} \ldots \lambda_{\ell(\lambda)}, \quad \sigma(\lambda)=\prod_{k \geq 1} m_{k} !, \quad z_{\lambda}=\sigma(\lambda) \rho(\lambda) .
$$


One can introduce some orderings on the set of diagrams with fixed $|\lambda|[10]$. One of them is the reverse lexicographical order: $\lambda$ precedes $\mu$ if the first non-vanishing difference $\lambda_{i}-\mu_{i}$ is positive. In this ordering $(n)$ comes first and $\left(1^{n}\right)$ comes last. It is a linear ordering. Another ordering is the natural partial ordering which is defined as follows:

$$
\lambda \geq \mu \Longleftrightarrow \lambda_{1}+\ldots+\lambda_{i} \geq \mu_{1}+\ldots+\mu_{i} \quad \text { for all } i \geq 1 .
$$

As soon as $|\lambda| \geq 6$ it is not a total ordering.

Schur functions. The general reference is [10]. Let $\mathbf{t}=\left\{t_{1}, t_{2}, t_{3}, \ldots\right\}$ be an infinite set of variables. The Schur polynomials $s_{\lambda}(\mathbf{t})$ labeled by Young diagrams $\lambda$ can be defined by the determinant formula

$$
s_{\lambda}(\mathbf{t})=\operatorname{det}_{i, j=1, \ldots, \ell(\lambda)} h_{\lambda_{i}-i+j}(\mathbf{t}),
$$

where the polynomials $h_{j}(\mathbf{t})$ are defined with the help of the generating series

$$
\exp \left(\sum_{k \geq 1} t_{k} z^{k}\right)=\sum_{k \geq 0} h_{k}(\mathbf{t}) z^{k}
$$

or, explicitly, $h_{k}(\mathbf{t})=\sum_{k_{1}+2 k_{2}+\ldots=k} \frac{t_{1}^{k_{1}}}{k_{1} !} \frac{t_{2}^{k_{2}}}{k_{2} !} \ldots=\sum_{l=1}^{k} \frac{1}{l !} \sum_{\substack{k_{1}, \ldots, k_{l} \geq 1 \\ k_{1}+\ldots+k_{l}=k}} t_{k_{1}} \ldots t_{k_{l}}$. The first few polynomials are $h_{1}(\mathbf{t})=t_{1}, h_{2}(\mathbf{t})=\frac{1}{2} t_{1}^{2}+t_{2}, h_{3}(\mathbf{t})=\frac{1}{6} t_{1}^{3}+t_{1} t_{2}+t_{3}, h_{4}(\mathbf{t})=\frac{1}{24} t_{1}^{4}+\frac{1}{2} t_{2}^{2}+$ $\frac{1}{2} t_{1}^{2} t_{2}+t_{1} t_{3}+t_{4}$. It is convenient to put $h_{0}(\mathbf{t})=1, h_{k}(\mathbf{t})=0$ for $k<0$ and $s_{\emptyset}(\mathbf{t})=1$. The functions $h_{k}$ are elementary Schur polynomials in the sense that for one-row diagrams $s_{(j)}(\mathbf{t})=h_{j}(\mathbf{t})$. Equation (4) is known as the Jacobi-Trudi identity.

We will need the Cauchy-Littlewood identity

$$
\sum_{\lambda} s_{\lambda}(\mathbf{t}) s_{\lambda}\left(\mathbf{t}^{\prime}\right)=\exp \left(\sum_{k \geq 1} k t_{k} t_{k}^{\prime}\right)
$$

where the sum is over all Young diagrams including the empty one. The both sides can be regarded as formal series in the variables $\mathbf{t}, \mathbf{t}^{\prime}$. Writing it in the form

$$
\sum_{\lambda} s_{\lambda}(\mathbf{y}) s_{\lambda}(\tilde{\partial})=\exp \left(\sum_{k \geq 1} y_{k} \partial_{t_{k}}\right)
$$

where $\tilde{\partial}=\left\{\partial_{t_{1}}, \frac{1}{2} \partial_{t_{2}}, \frac{1}{3} \partial_{t_{3}}, \ldots\right\}$ and applying to $s_{\mu}(\mathbf{t})$, we get the relation

$$
\left.s_{\lambda}(\tilde{\partial}) s_{\mu}(\mathbf{t})\right|_{\mathbf{t}=0}=\delta_{\lambda \mu}
$$

which reflects the orthonormality of the Schur functions.

The Schur functions are usually regarded as symmetric functions of the variables $x_{i}$ defined by $k t_{k}=\sum_{i} x_{i}^{k}$. In terms of the variables $x_{i}(i=1, \ldots, N, N \geq \ell(\lambda))$,

$$
s_{\lambda}(\mathbf{t})=s_{\lambda}\left(\left\{x_{i}\right\}_{N}\right)=\frac{\operatorname{det}_{1 \leq i, j \leq N}\left(x_{i}^{N+\lambda_{j}-j}\right)}{\operatorname{det}_{1 \leq i, j \leq N}\left(x_{i}^{N-j}\right)}, \quad t_{k}=\frac{1}{k} \sum_{i} x_{i}^{k} .
$$

It can be proved [10] that the Schur polynomials form an orthonormal basis in the space of symmetric functions. 
Dual bases in the space of polynomials. Let us consider the linear space $\mathcal{P}$ of polynomials in the variables $\mathbf{t}=\left\{t_{1}, t_{2}, t_{3}, \ldots\right\}$. The basis vectors are naturally labeled by Young diagrams with basis polynomials $v_{\lambda}(\mathbf{t})$ being quasi-homogeneous in the sense that $v_{\lambda}\left(a t_{1}, a^{2} t_{2}, a^{3} t_{3}, \ldots\right)=a^{|\lambda|} v_{\lambda}\left(t_{1}, t_{2}, t_{3}, \ldots\right)$. By definition, the polynomial labelled by the empty diagram $\emptyset$ is $v_{\emptyset}(\mathbf{t})=1$. One may choose such a basis in many different ways. The simplest example is the usual monomial basis

$$
t_{\lambda}(\mathbf{t}):=t_{\lambda_{1}} t_{\lambda_{2}} \ldots t_{\lambda_{\ell}}=\prod_{i \geq 1} t_{i}^{m_{i}(\lambda)}
$$

Other examples are the basis $h_{\lambda}(\mathbf{t})=h_{\lambda_{1}} h_{\lambda_{2}} \ldots h_{\lambda_{\ell}}$ and the Schur polynomial basis $s_{\lambda}(\mathbf{t})$. Having in mind the interpretation of polynomials of $t_{k}$ as symmetric functions of the variables $x_{i}$ discussed above, we will call polynomials from the space $\mathcal{P}$ symmetric functions (although they are not symmetric w.r.t. the variables $t_{k}$ ). Another standard basis in $\mathcal{P}$ is the basis of monomial symmetric functions $m_{\lambda}=m_{\lambda}(\mathbf{t})$ which are easily defined in terms of the variables $x_{1}, x_{2}, \ldots, x_{n}$ provided $n \geq \ell(\lambda)$ (it is implied that $\lambda_{j}=0$ if $j>\ell(\lambda))$ :

$$
m_{\lambda}\left(x_{1}, x_{2}, \ldots, x_{n}\right)=\frac{1}{(n-\ell(\lambda)) ! \sigma(\lambda)} \sum_{P \in S_{n}} x_{1}^{\lambda_{P(1)}} x_{2}^{\lambda_{P(2)}} \ldots x_{n}^{\lambda_{P(n)}} .
$$

The normalization factor is chosen in such a way that the function $m_{\lambda}(\mathbf{t})$ does not depend on $n$ if $n \geq \ell(\lambda)$. For example, $m_{(k)}(\mathbf{t})=\sum_{j} x_{j}^{k}=k t_{k}$. The first few functions $m_{\lambda}$ are:

$$
\begin{aligned}
& m_{(1)}(\mathbf{t})=t_{1} \\
& m_{(2)}(\mathbf{t})=2 t_{2}, \quad m_{\left(1^{2}\right)}(\mathbf{t})=\frac{1}{2} t_{1}^{2}-t_{2} \\
& m_{(3)}(\mathbf{t})=3 t_{3}, \quad m_{(21)}(\mathbf{t})=2 t_{2} t_{1}-3 t_{3}, \quad m_{\left(1^{3}\right)}(\mathbf{t})=\frac{1}{6} t_{1}^{3}-t_{2} t_{1}+t_{3}
\end{aligned}
$$

It is also convenient to keep the standard notation $p_{k}$ for the power sums $p_{k}=k t_{k}$ and the corresponding basis $p_{\lambda}(\mathbf{t})=\rho(\lambda) t_{\lambda}$.

One can introduce the scalar product in the space $\mathcal{P}$ as follows:

$$
\left\langle u_{\lambda}, v_{\mu}\right\rangle=\left.u_{\lambda}(\tilde{\partial}) v_{\mu}(\mathbf{t})\right|_{\mathbf{t}=0} .
$$

This definition is symmetric, i.e., $\left\langle u_{\lambda}, v_{\mu}\right\rangle=\left\langle v_{\mu}, u_{\lambda}\right\rangle$. This scalar product coincides with the one from [10] defined axiomatically in the space of symmetric functions. In particular, we have: $\left\langle p_{\lambda}, p_{\mu}\right\rangle=z_{\lambda} \delta_{\lambda \mu}$, where,$\left\langle s_{\lambda}, s_{\mu}\right\rangle=\delta_{\lambda \mu}$ and $\left\langle h_{\lambda}, m_{\mu}\right\rangle=\delta_{\lambda \mu}$. If $\left\langle u_{\lambda}, v_{\mu}\right\rangle=\delta_{\lambda \mu}$ for all $\lambda, \mu$, we say that $u_{\lambda}, v_{\lambda}$ are dual bases.

Lemma 1.1 Let $u_{\lambda}(\mathbf{t}), v_{\lambda}(\mathbf{t})$ be any pair of dual bases in $\mathcal{P}$, and $g(\mathbf{t})$ be any function real-analytic around the point $\mathbf{t}=0$, then the Taylor series at $\mathbf{t}=0$ can be represented in the form

$$
g(\mathbf{t})=\left.\sum_{\lambda} u_{\lambda}(\tilde{\partial}) g\left(\mathbf{t}^{\prime}\right)\right|_{\mathbf{t}^{\prime}=0} v_{\lambda}(\mathbf{t})
$$

The proof is obvious. 


\section{The $\hbar$-KP hierarchy for the tau-function}

Hereafter we work with the $\hbar$-formulation [22] of the KP hierarchy and call it the $\hbar$-KP hierarchy. Let $\tau=\tau\left(t_{1}, t_{2}, t_{3}, \ldots\right)=\tau(\mathbf{t})$ be the tau-function of the hierarchy. It depends on the infinite set of time variables $\mathbf{t}=\left\{t_{1}, t_{2}, \ldots\right\}$. It also contains $\hbar$ as a parameter but we will not write it explicitly.

\subsection{Hirota equations for the tau-function}

The $\hbar$-KP hierarchy can be represented in different equivalent forms. We start with the Hirota bilinear equations for the tau-function. They can be encoded in a functional relation. Below we use the notation

$$
\tau^{\left[z_{1}, \ldots, z_{m}\right]}(\mathbf{t})=\tau\left(\mathbf{t}+\hbar \sum_{i=1}^{m}\left[z_{i}^{-1}\right]\right)=e^{\hbar\left(D\left(z_{1}\right)+\ldots+D\left(z_{m}\right)\right)} \tau
$$

where

$$
\mathbf{t} \pm \hbar\left[z^{-1}\right]:=\left\{t_{1} \pm \hbar z^{-1}, t_{2} \pm \frac{\hbar}{2} z^{-2}, t_{3} \pm \frac{\hbar}{3} z^{-3}, \ldots,\right\}
$$

and the differential operator $D(z)$ is defined by

$$
D(z)=\sum_{k \geq 1} \frac{z^{-k}}{k} \partial_{k}
$$

Hereafter we abbreviate $\partial_{k}=\partial / \partial t_{k}$.

The Hirota functional relation for the tau-function reads

$$
\left(z_{1}-z_{2}\right) \tau^{\left[z_{1}, z_{2}\right]} \tau^{\left[z_{3}\right]}+\left(z_{2}-z_{3}\right) \tau^{\left[z_{2}, z_{3}\right]} \tau^{\left[z_{1}\right]}+\left(z_{3}-z_{1}\right) \tau^{\left[z_{3}, z_{1}\right]} \tau^{\left[z_{2}\right]}=0
$$

It is to be fulfilled for all $z_{1}, z_{2}, z_{3}$. By tau-function (of the $\hbar$-KP hierarchy) we mean any solution to this equation. Differential equations of the hierarchy are obtained by expanding this equation in powers of $z_{1}, z_{2}, z_{3}$. Another form of the functional relation for the $\tau$-function is

$$
\hbar \partial_{1} \log \frac{\tau^{\left[z_{1}\right]}}{\tau^{\left[z_{2}\right]}}=\left(z_{2}-z_{1}\right)\left(\frac{\tau^{\left[z_{1}, z_{2}\right]} \tau}{\tau^{\left[z_{1}\right]} \tau^{\left[z_{2}\right]}}-1\right)
$$

which is sometimes called the differential Fay identity.

Proposition 2.1 Equations (12) and (13) are equivalent.

Proof. Eq. (13) follows from eq. (12) in the limit $z_{3} \rightarrow \infty$. Eq. (12) can be obtained from (13) by summing the equations written for the pairs $\left\{z_{1}, z_{2}\right\},\left\{z_{2}, z_{3}\right\},\left\{z_{3}, z_{1}\right\}$.

Proposition 2.2 The tau-function $\tau=\tau(\mathbf{t})$ of the $\hbar$-KP hierarchy satisfies the equations

$$
\prod_{1 \leq i<j \leq m}\left(z_{j}-z_{i}\right) \cdot \tau^{\left[z_{1}, \ldots, z_{m}\right]} \tau^{m-1}=\operatorname{det}_{1 \leq j, k \leq m}\left(\left(z_{j}-\hbar \partial_{1}\right)^{k-1} \tau^{\left[z_{j}\right]}\right)
$$

for any $m \geq 2$ and any $z_{1}, \ldots, z_{m}$. 
Proof. At $m=2$ equation (14) reads

$$
\left(z_{2}-z_{1}\right) \tau^{\left[z_{1}, z_{2}\right]} \tau=\left|\begin{array}{cc}
\tau^{\left[z_{1}\right]} & \left(z_{1}-\hbar \partial_{1}\right) \tau^{\left[z_{1}\right]} \\
\tau^{\left[z_{2}\right]} & \left(z_{2}-\hbar \partial_{1}\right) \tau^{\left[z_{2}\right]}
\end{array}\right|
$$

which is (13). The rest of the proof is induction in $m$. See Appendix A for details.

Equation (14) was first suggested in [1].

\subsection{Formal solution for the tau-function}

Let us introduce the differential operators

$$
\partial_{k}^{\hbar}=\frac{k}{\hbar} h_{k}(\hbar \tilde{\partial})=\partial_{k}+\hbar \sum_{l=1}^{k-1} \frac{k \partial_{l} \partial_{k-l}}{2 l(k-l)}+O\left(\hbar^{2}\right),
$$

where $h_{k}(\mathbf{t})$ are the elementary Schur polynomials. These operators are " $\hbar$-deformations" of the partial derivatives in the sense that $\partial_{k}^{\hbar=0}=\partial_{k}$. We call them $\hbar$-deformed partial derivatives. Their properties are studied below in the next section.

In the KP theory the first variable, $t_{1}$, is distinguished. Having this in mind, we will treat the tau-function depending on $t_{i}$ as a result of the evolution of $\tau(x ; \mathbf{0})$ according to the KP flows: $\tau(x ; \mathbf{0}) \rightarrow \tau(x ; \mathbf{t})=f(x) \hat{\tau}\left(x+t_{1}, t_{2}, t_{3}, \ldots\right)$. Our aim is to represent it as a formal series in the $t_{i}$ 's given $\tau(x ; \mathbf{0})$ and some Cauchy-like data to be specified below.

With the help of the operators $\partial_{i}^{\hbar}$ we now define modified Cauchy data which are necessary for constructing the formal solution. We call them the Cauchy-like data.

Definition 2.1 The Cauchy-like data of a solution $\tau(x ; \mathbf{t})$ to the $\hbar$-KP hierarchy is the set of functions $\tau(x ; \mathbf{0}),\left.\partial_{k}^{\hbar} \tau(x ; \mathbf{t})\right|_{\mathbf{t}=0}, k \geq 1$.

The following theorem asserts that there exists a formal solution for arbitrary infinitely differentiable Cauchy-like data. It also provides its constructive representation assuming $\hbar \neq 0$.

Theorem 2.1 Let $\tau(x, \mathbf{t})=f(x) \hat{\tau}\left(x+t_{1}, t_{2}, \ldots\right)$ be a tau-function of the $\hbar-K P$ hierarchy with respect to the variables $t_{j}$, with $\tau(x, \mathbf{0})$ being an infinitely differentiable function of $x$. Then the coefficients of the series

$$
\tau(x ; \mathbf{t})=\sum_{\lambda} c_{\lambda}(x) s_{\lambda}(\mathbf{t} / \hbar)
$$

are connected by the relations

$$
c_{\lambda}(x)=\left(c_{0}(x)\right)^{1-\ell(\lambda)} \operatorname{det}_{1 \leq i, j \leq \ell(\lambda)}\left[\sum_{k=0}^{j-1}(-\hbar)^{k}\left(\begin{array}{c}
j-1 \\
k
\end{array}\right) \partial_{x}^{k} c_{\lambda_{i}-i+j-k}(x)\right]
$$


where $\left(\begin{array}{c}j-1 \\ k\end{array}\right)=\frac{(j-1) !}{k !(j-1-k) !}$ is the binomial coefficient, $c_{0}(x)=c_{\emptyset}(x), c_{k}(x)=c_{(k)}(x)$ and $c_{1}=\partial_{x} c_{0}-c_{0} \partial_{x} \log f$.

Conversely, let $\hbar \neq 0$ and $c_{k}(x), k=0,1,2, \ldots$, be arbitrary infinitely differentiable functions of $x$ (with $c_{0}(x)$ being not identically 0 ); for any Young diagram $\lambda$ define the coefficients $c_{\lambda}(x)$ by (17). Then the series (16) is a formal solution to the $\hbar-K P$ hierarchy $(\hbar \neq 0)$ with the Cauchy-like data

$$
\tau(x ; \mathbf{0})=c_{0}(x),\left.\quad \partial_{k}^{\hbar} \tau(x ; \mathbf{t})\right|_{\mathbf{t}=0}=\frac{k}{\hbar} c_{k}(x), \quad k \geq 1 .
$$

Proof. To prove the first part of the theorem, we note that equations (14) for the taufunction $\tau(x ; \mathbf{t})$ can be written in the form

$$
\prod_{1 \leq a<b \leq m}\left(z_{b}-z_{a}\right) \cdot \tau^{\left[z_{1}, \ldots, z_{m}\right]}(x ; \mathbf{t}) \tau^{m-1}(x ; \mathbf{t})=\operatorname{det}_{1 \leq i, j \leq m}\left(\left(z_{i}-\hbar \partial_{x}\right)^{j-1} \tau^{\left[z_{i}\right]}(x ; \mathbf{t})\right)
$$

(as is easy to see, derivatives of $f$ coming from $\partial_{x}^{l}(f \tau)$ cancel in the determinant in the right hand side after taking proper linear combinations of columns). The idea is to substitute (16) into this equation and equate the coefficients of the $z_{i}$-expansion of both sides at $\mathbf{t}=0$. Let us rewrite (18) in the form convenient for expanding in powers of $z_{i}^{-1}$ :

$$
\tau^{\left[z_{1}, \ldots, z_{m}\right]}(x ; \mathbf{t})=\frac{\operatorname{det}\left(z_{i}^{j-m}\left(1-\hbar z_{i}^{-1} \partial_{x}\right)^{j-1} \tau^{\left[z_{i}\right]}(x ; \mathbf{t})\right)}{\operatorname{det}\left(z_{i}^{j-m}\right) \tau^{m-1}(x ; \mathbf{t})}, \quad m \geq 2 .
$$

It follows from the Cauchy-Littlewood identity that the expansion of the left hand side is

$$
e^{\hbar\left(D\left(z_{1}\right)+\ldots+D\left(z_{m}\right)\right)} \tau(x ; \mathbf{t})=\sum_{\lambda} s_{\lambda}\left(\left\{z_{i}^{-1}\right\}\right) s_{\lambda}(\hbar \tilde{\partial}) \tau(x ; \mathbf{t})
$$

(here only terms with $\ell(\lambda) \leq m$ are non-zero). The numerator of the right hand side is $\underset{1 \leq i, j \leq m}{\operatorname{det}}\left(\sum_{l \geq 0} Z_{i l} M_{l j}\right)$, where $Z_{i l}=z_{i}^{-l}$ is the rectangular semi-infinite $m \times \mathbb{Z}_{\geq 0}$ matrix and

$$
M_{l j}=\sum_{a=0}^{j-1}(-\hbar)^{a}\left(\begin{array}{c}
j-1 \\
a
\end{array}\right) \partial_{x}^{a} h_{l+j-a-m}(\hbar \tilde{\partial}) \tau(x ; \mathbf{t})
$$

is the rectangular semi-infinite $\mathbb{Z}_{\geq 0} \times m$ matrix (we put $c_{k}=0$ at $k<0$ ). Application of the Cauchy-Binet formula gives

$$
\operatorname{det}_{1 \leq i, j \leq m}\left(\sum_{l \geq 0} Z_{i l} M_{l j}\right)=\sum_{l_{1}>l_{2}>\ldots>l_{m} \geq 0} \operatorname{det}_{i j}\left(z_{i}^{-l_{j}}\right) \cdot \operatorname{det} M_{l_{j} k}
$$

Setting $l_{j}=m+\lambda_{j}-j$, we can represent the multiple sum as summation over Young diagrams with rows $\lambda_{i}$. Using (7), we see that the expansion of the right hand side is

$$
\tau(x, \mathbf{t})^{1-m} \sum_{\lambda} s_{\lambda}\left(\left\{z_{i}^{-1}\right\}\right) \operatorname{det}_{i j}\left[\sum_{a=0}^{j-1}(-\hbar)^{a}\left(\begin{array}{c}
j-1 \\
a
\end{array}\right) \partial_{x}^{a} h_{\lambda_{i}-i+j-a}(\hbar \tilde{\partial}) \tau(x ; \mathbf{t})\right]
$$

Comparing with (20), it remains to put $\mathbf{t}=\mathbf{0}$ and use the orthonormality of the Schur functions. 
Now let us prove that the series (16) with $c_{\lambda}$ built from arbitrary $c_{k}$ according to (17) solves the Hirota equations (12) or (13). First we repeat the above derivation in reverse order to prove that equations (18) hold for any $z_{1}, \ldots, z_{m}$ and $m \geq 2$ at $\mathbf{t}=0$. The next step is to deduce that this set of relations for "initial values" of $\tau^{\left[z_{1}, \ldots, z_{m}\right]}$ is equivalent to

$$
\left(z_{2}-z_{1}\right) \tau^{\left[z_{1}, z_{2}, z_{3}, \ldots, z_{m}\right]}(x ; \mathbf{0}) \tau^{\left[z_{3}, \ldots, z_{m}\right]}(x ; \mathbf{0})=\left|\begin{array}{ll}
\tau^{\left[z_{1}, z_{3}, \ldots, z_{m}\right]}(x ; \mathbf{0}) & \left(z_{1}-\hbar \partial_{x}\right) \tau^{\left[z_{1}, z_{3}, \ldots, z_{m}\right]}(x ; \mathbf{0}) \\
\tau^{\left[z_{2}, z_{3}, \ldots, z_{m}\right]}(x ; \mathbf{0}) & \left(z_{2}-\hbar \partial_{x}\right) \tau^{\left[z_{2}, z_{3}, \ldots, z_{m}\right]}(x ; \mathbf{0})
\end{array}\right|
$$

(which is the Jacobi identity for the matrix $N_{i j}=\left(z_{i}-\partial_{x}\right)^{j-1} \tau^{\left[z_{i}\right]}(x ; \mathbf{0})$, see appendix A for details). In its turn, this latter relation, being valid for all $z_{1}, \ldots, z_{m}$ and $m \geq 2$, is equivalent to

$$
\left(z_{2}-z_{1}\right) \tau^{\left[z_{1}, z_{2}\right]}(x ; \mathbf{t}) \tau(x ; \mathbf{t})=\left|\begin{array}{ll}
\tau^{\left[z_{1}\right]}(x ; \mathbf{t}) & \left(z_{1}-\hbar \partial_{x}\right) \tau^{\left[z_{1}\right]}(x ; \mathbf{t}) \\
\tau^{\left[z_{2}\right]}(x ; \mathbf{t}) & \left(z_{2}-\hbar \partial_{x}\right) \tau^{\left[z_{2}\right]}(x ; \mathbf{t})
\end{array}\right|
$$

for any $\mathbf{t}$. This can be formally justified by noticing that if $\left.e^{\hbar\left(D\left(z_{1}\right)+\ldots+D\left(z_{k}\right)\right)} \mathcal{F}(\mathbf{t})\right|_{\mathbf{t}=0}=0$ for some $\mathcal{F}(\mathbf{t})$ for all $z_{1}, \ldots, z_{k}$ and $k \geq 0$, then $\left.\sum_{\lambda} s_{\lambda}\left(\left\{z_{i}^{-1}\right\}\right) s_{\lambda}(\tilde{\partial}) \mathcal{F}(\mathbf{t})\right|_{\mathbf{t}=0}=0$, whence $\left.s_{\lambda}(\tilde{\partial}) \mathcal{F}(\mathbf{t})\right|_{\mathbf{t}=0}=0$ for all $\lambda$ which means that $\mathcal{F}(\mathbf{t}) \equiv 0$ as a formal series. Multiplying both sides of equation (21) by $\frac{\tau^{\left[z_{3}\right]}}{\tau^{\left[z_{1}\right]} \tau^{\left[z_{2}\right]}}$ and summing the equations obtained in this way for all cyclic permutations of $\left\{z_{1}, z_{2}, z_{3}\right\}$, we get the Hirota equation (12) which, by Proposition 2.2, is equivalent to (13). The latter equation has the form (21) with $\partial_{x} \rightarrow \partial_{1}$, so the assertion is proved.

It remains to show that the tau-function represented by the series (16) does have the form $f(x) \hat{\tau}\left(x+t_{1} ; t_{2}, \ldots\right)$, i.e., it essentially depends on $x+t_{1}$. For this we notice that the above argument implies that

$$
\left(\partial_{x}-\partial_{1}\right)\left(\log \tau\left(x ; \mathbf{t}+\hbar\left[z^{-1}\right]\right)-\log \tau(x ; \mathbf{t})\right)=0
$$

for all $\mathbf{t}, z$. From this it then follows that $\varphi:=\left(\partial_{x}-\partial_{1}\right) \log \tau(x ; \mathbf{t})$ does not depend on t. Indeed, we have

$$
0=\varphi\left(\mathbf{t}+\hbar\left[z^{-1}\right]\right)-\varphi(\mathbf{t})=\hbar \partial_{1} \varphi z^{-1}+\frac{1}{2}\left(\hbar^{2} \partial_{1}^{2} \varphi+\hbar \partial_{2} \varphi\right) z^{-2}+\ldots
$$

from which we can conclude that $\partial_{1} \varphi=\partial_{2} \varphi=\ldots=0$. Therefore, $\left(\partial_{x}-\partial_{1}\right) \log \tau(x ; \mathbf{t})=$ $\varphi(x)$. A simple calculation at $\mathbf{t}=\mathbf{0}$ shows that $\varphi(x)=\partial_{x} \log c_{0}(x)-\frac{c_{1}(x)}{c_{0}(x)}$.

Remark 2.1 Putting $c_{1}(x)=\partial_{x} c_{0}(x)$, one obtains tau-functions $\tau\left(x+t_{1}, t_{2}, \ldots\right)$ whose $t_{1}$-evolution is equivalent to the shift of $x$.

Remark 2.2 Theorem [2.1 has been basically proven in [1], where equation [17) (with $\hbar=1$ ) has appeared as a functional equation for commuting transfer matrices of the quantum Gaudin model. 


\section{The $\hbar$-KP hierarchy for the $F$-function}

\subsection{Hirota equations for the $F$-function}

For many applications in physics and mathematics one needs to deal with logarithm of the tau-function rather than with the tau-function itself. It is possible to represent the Hirota equations as equations for the logarithm. Let us introduce the $F$-function as

$$
F(x ; \mathbf{t})=\hbar^{2} \log \tau(x ; \mathbf{t}) .
$$

Representing shifts of the arguments in (12) as action of exponential of the differential operators $D\left(z_{i}\right)$ to the function $F$, we can rewrite (12) in terms of $F$ :

$$
\begin{aligned}
& \left(z_{1}-z_{2}\right) \exp \left[\hbar^{-2} e^{\hbar\left(D\left(z_{1}\right)+D\left(z_{2}\right)\right)} F\right] \exp \left[\hbar^{-2} e^{\hbar D\left(z_{3}\right)} F\right] \\
& +\left(z_{2}-z_{3}\right) \exp \left[\hbar^{-2} e^{\hbar\left(D\left(z_{2}\right)+D\left(z_{3}\right)\right)} F\right] \exp \left[\hbar^{-2} e^{\hbar D\left(z_{1}\right)} F\right] \\
& \quad+\left(z_{3}-z_{1}\right) \exp \left[\hbar^{-2} e^{\hbar\left(D\left(z_{1}\right)+D\left(z_{3}\right)\right)} F\right] \exp \left[\hbar^{-2} e^{\hbar D\left(z_{2}\right)} F\right]=0
\end{aligned}
$$

One can represent (23) in a more suggestive form of a non-linear difference equation. Let us introduce the difference operator

$$
\Delta(z)=\frac{e^{\hbar D(z)}-1}{\hbar}
$$

It can be easily checked that equation (23) acquires the form

$$
\left(z_{1}-z_{2}\right) e^{\Delta\left(z_{1}\right) \Delta\left(z_{2}\right) F}+\left(z_{2}-z_{3}\right) e^{\Delta\left(z_{2}\right) \Delta\left(z_{3}\right) F}+\left(z_{3}-z_{1}\right) e^{\Delta\left(z_{3}\right) \Delta\left(z_{1}\right) F}=0 .
$$

Tending $z_{3} \rightarrow \infty$ we get the differential Fay identity (13) in terms of $F$ :

$$
e^{\Delta\left(z_{1}\right) \Delta\left(z_{2}\right) F}=1-\frac{\Delta\left(z_{1}\right) \partial_{1} F-\Delta\left(z_{2}\right) \partial_{1} F}{z_{1}-z_{2}} .
$$

For $F(x ; \mathbf{t})=\phi(x)+\hat{F}\left(x+t_{1}, t_{2}, t_{3}, \ldots\right)$ we can also write this as

$$
e^{\Delta\left(z_{1}\right) \Delta\left(z_{2}\right) F}=1-\frac{\Delta\left(z_{1}\right) \partial_{x} F-\Delta\left(z_{2}\right) \partial_{x} F}{z_{1}-z_{2}}
$$

In the form (25) the equation is suitable for the $\hbar$-expansion as $\hbar \rightarrow 0$. The $\hbar$ expansion of the $\hbar-\mathrm{KP}$ hierarchy was investigated in [23, 24] (see also [25]). An important class of solutions is distinguished by the assumption that the function $F$ has a regular $\hbar$-expansion:

$$
F=F^{(0)}+\hbar F^{(1)}+\hbar^{2} F^{(2)}+O\left(\hbar^{3}\right) .
$$

The case $\hbar=0$ is called the zero dispersion (or dispersionless) limit. In this limit we have $\lim _{\hbar \rightarrow 0} \Delta(z)=D(z)$ and equation (26) becomes the familiar dispersionless Hirota equation for $F^{(0)}$. 


\subsection{The $\hbar$-KP hierarchy in an unfolded form}

Another useful representation of the KP hierarchy is an "unfolded" form suggested in [3, 17]. The notation $\partial:=\partial_{x}, F_{k}:=\partial_{k} F, F_{k l}:=\partial_{k} \partial_{l} F$, etc is convenient. The following theorem can be proven in the same way as [17, Theorem 2], with minor modifications keeping track of the $\hbar$-dependence.

Theorem 3.1 There exist universal rational coefficients $R_{i j}^{n}\left(\begin{array}{ccc}s_{1} & \ldots & s_{n} \\ r_{1} & \ldots & r_{n}\end{array}\right)$ such that

$$
F_{k l}=\sum_{n \geq 1} \sum \hbar^{\sum_{i}\left(r_{i}-1\right)} R_{k l}^{n}\left(\begin{array}{ccc}
s_{1} & \ldots & s_{n} \\
r_{1} & \ldots & r_{n}
\end{array}\right) \partial^{r_{1}} F_{s_{1}} \ldots \partial^{r_{n}} F_{s_{n}}
$$

Here the second sums are taken over all matrices $\left(\begin{array}{lll}s_{1} & \ldots & s_{n} \\ r_{1} & \ldots & r_{n}\end{array}\right)$ such that $s_{i}, r_{i} \geq 1$ and $\sum_{i=1}^{n}\left(s_{i}+r_{i}\right)=k+l$

Equations (29) represent the KP hierarchy in the unfolded form. They express $F_{k l}$ with $k, l>1$ as certain universal polynomials of $\partial^{r} F_{j}$ 's with $r, j \geq 1$. The coefficients of these polynomials are fixed rational numbers which do not depend on a particular solution.

Corollary 3.1 For any integer $m \geq 2$ there exist universal rational coefficients $R_{k_{1} \ldots k_{m}}^{n}\left(\begin{array}{ccc}s_{1} & \ldots & s_{n} \\ r_{1} & \ldots & r_{n}\end{array}\right)$ such that

$$
F_{k_{1} \ldots k_{m}}=\sum_{n \geq 1} \sum \hbar^{\sum_{i}\left(r_{i}-1\right)+2-m} R_{k_{1} \ldots k_{m}}^{n}\left(\begin{array}{ccc}
s_{1} & \ldots & s_{n} \\
r_{1} & \ldots & r_{n}
\end{array}\right) \partial^{r_{1}} F_{s_{1}} \ldots \partial^{r_{n}} F_{s_{n}}
$$

The second sums are taken over all matrices $\left(\begin{array}{lll}s_{1} & \ldots & s_{n} \\ r_{1} & \ldots & r_{n}\end{array}\right)$ such that $s_{i}, r_{i} \geq 1$ with the conditions $\sum_{i=1}^{n}\left(s_{i}+r_{i}\right)=\sum_{i=1}^{n} k_{i}, \sum_{i=1}^{n} r_{i} \geq n+m-2$.

Proof. The proof consists in induction on $m$ using the Leibniz rule.

It follows from the Corollary that the quantities $F_{j}, j \geq 1$ restricted to zero values of $t_{k}$ with $k \geq 2$ define the solution up to a constant. In this way, one produces a unique formal Taylor series in $t_{j}$ 's which reconstructs a formal solution to the KP hierarchy from the Cauchy data $f_{i}(x):=\left.\partial_{i} F(x ; \mathbf{t})\right|_{t_{1}=t_{2}=\ldots=0}$. In general, the functions $f_{i}$ can be Taylor series in $\hbar$.

The following theorem can be proven in the same way as Theorem 4.2 from [18.

Theorem 3.2 If $\sum_{i=1}^{n}\left(r_{i}-1\right)-m \equiv 1(\bmod 2)$, then $R_{k_{1} \ldots k_{m}}^{n}\left(\begin{array}{lll}s_{1} & \ldots & s_{n} \\ r_{1} & \ldots & r_{n}\end{array}\right)=0$. 
We see that equations (30) contain even powers of $\hbar$ only. Therefore, if the Cauchy data $f_{i}(x)$ are series in $\hbar^{2}$, then so is the formal solution. In particular, the formal solution is expanded in only even powers of $\hbar$ if the Cauchy data are $\hbar$-independent.

At $\hbar=0$ only the terms with $r_{i}=1$ survive in (29) and these equations yield the polynomial expressions of $F_{k l}^{(0)}$ with $k, l>1$ through $F_{1 j}^{(0)}$ 's. In this case there is a relatively simple recurrence formula for the coefficients, see [17]. In general any explicit combinatorial description of the coefficients $R_{k_{1} \ldots k_{m}}^{n}$ is not available.

In the rest part of the paper we show that some closed description of expansion coefficients can be achieved for non-zero $\hbar$, too, by a re-summation of the Taylor series and passing to another basis in the space of polynomials in $t_{k}$ 's. We will exploit the formal similarity of the Hirota equation (26) with its dispersionless limit.

\subsection{The “ $\hbar$-deformed partial derivatives" $\partial_{i}^{\hbar}$}

Let us recall the definition of the " $\hbar$-deformed partial derivatives" $\partial_{i}^{\hbar}$ introduced in (15):

$$
\partial_{k}^{\hbar}=\hbar^{-1} k h_{k}(\hbar \tilde{\partial}), \quad \tilde{\partial} \equiv\left\{\partial_{1}, \frac{1}{2} \partial_{2}, \frac{1}{3} \partial_{3}, \ldots\right\}
$$

The first few are: $\partial_{1}^{\hbar}=\partial_{1}, \partial_{2}^{\hbar}=\partial_{2}+\hbar \partial_{1}^{2}, \partial_{3}^{\hbar}=\partial_{3}+\frac{3}{2} \hbar \partial_{1} \partial_{2}+\frac{1}{2} \hbar^{2} \partial_{1}^{3}$. It is convenient to put $\partial_{0}^{\hbar}=1$. Here we discuss these operators in some detail.

The general explicit formula is

$$
\partial_{k}^{\hbar}=\sum_{l=1}^{k} \frac{\hbar^{l-1} k}{l !} \sum_{\substack{k_{1}, \ldots, k_{l} \geq 1 \\ k_{1}+\ldots+k_{l}=k}} \frac{\partial_{k_{1}} \ldots \partial_{k_{l}}}{k_{1} \ldots k_{l}}
$$

There is also a determinant representation which can be extracted from [10]:

$$
\partial_{n}^{\hbar}=\frac{1}{(n-1) !}\left|\begin{array}{cccccc}
\partial_{1} & -1 & 0 & 0 & \ldots & 0 \\
\partial_{2} & \hbar \partial_{1} & -2 & 0 & \ldots & 0 \\
\partial_{3} & \hbar \partial_{2} & \hbar \partial_{1} & -3 & \ldots & 0 \\
\ldots & \ldots & \ldots & \ldots & \ldots & \ldots \\
\partial_{n-1} & \hbar \partial_{n-2} & \hbar \partial_{n-3} & \hbar \partial_{n-4} & \ldots & -(n-1) \\
\partial_{n} & \hbar \partial_{n-1} & \hbar \partial_{n-2} & \hbar \partial_{n-3} & \ldots & \hbar \partial_{1}
\end{array}\right|
$$

The operators $\partial_{k}^{\hbar}$ have rather special properties. In particular, they satisfy the following generalized Leibniz rule.

Lemma 3.1 The differential operators $\partial_{k}^{\hbar}$ satisfy the generalized Leibniz rule:

$$
\partial_{k}^{\hbar} \prod_{i=1}^{n} f_{i}=\sum_{\substack{k_{1}, \ldots, k_{n} \geq 0 \\ k_{1}+\ldots+k_{n}=k}} \frac{\hbar^{\nu\left(k_{1}, \ldots, k_{n}\right)-1} k}{\left[k_{1} \ldots k_{n}\right]} \partial_{k_{1}}^{\hbar} f_{1} \partial_{k_{2}}^{\hbar} f_{2} \ldots \partial_{k_{n}}^{\hbar} f_{n}
$$

where $\nu\left(k_{1}, \ldots, k_{n}\right)$ is the number of nonzero $k_{i}$ 's in the sequence $k_{1}, k_{2}, \ldots, k_{n}$ and $\left[k_{1} \ldots k_{n}\right]:=\prod_{i=1}^{n} \max \left\{k_{i}, 1\right\}$ is their product. 
Proof. Expanding the both sides of the obvious identity $e^{\hbar D(z)} \prod_{i=1}^{n} f_{i}=\prod_{i=1}^{n}\left(e^{\hbar D(z)} f_{i}\right)$ in powers of $z$, we get:

$$
\sum_{k \geq 0} z^{-k} \mathrm{~h}_{k} \prod_{i=1}^{n} f_{i}=\sum_{k_{1}, \ldots, k_{n} \geq 0}\left(z^{-k_{1}} \mathrm{~h}_{k_{1}} f_{1}\right) \ldots\left(z^{-k_{n}} \mathrm{~h}_{k_{n}} f_{n}\right),
$$

where we have put $\mathrm{h}_{k}:=h_{k}(\hbar \tilde{\partial})$ for brevity. Equating coefficients in front of powers of $z$ in the both sides, we obtain the identity

$$
\mathrm{h}_{k} \prod_{i=1}^{n} f_{i}=\sum_{\substack{k_{1}, \ldots, k_{n} \geq 0 \\ k_{1}+\ldots,+k_{n}=k}}\left(\mathrm{~h}_{k_{1}} f_{1}\right) \ldots\left(\mathrm{h}_{k_{n}} f_{n}\right)
$$

Being rewritten in terms of the operators $\partial_{k}^{\hbar}$, it coincides with (34).

In the limit $\hbar \rightarrow 0$ (34) becomes the usual Leibniz rule for the partial derivatives $\partial_{k}$.

\subsection{The $\hbar$-KP hierarchy in terms of $\partial_{i}^{\hbar}$}

Following [15], we define the combinatorial constants $\tilde{P}_{i j}\left(s_{1}, \ldots, s_{m}\right)$ to be the number of sequences of positive integers $\left(i_{1}, \ldots, i_{m}\right),\left(j_{1}, \ldots, j_{m}\right)$ such that $i_{1}+\ldots+i_{m}=i$, $j_{1}+\ldots+j_{m}=j$ and $s_{k}=i_{k}+j_{k}-1$. Put

$$
P_{i j}\left(s_{1}, \ldots, s_{m}\right)=\frac{(-1)^{m+1} i j}{m s_{1} \ldots s_{m}} \tilde{P}_{i j}\left(s_{1}, \ldots, s_{m}\right) .
$$

Lemma 3.2 The $\hbar$-KP hierarchy (26) is equivalent to the system of equations

$$
\partial_{i}^{\hbar} \partial_{j}^{\hbar} F=\sum_{m \geq 1} \sum_{\substack{s_{1}, \ldots, s_{m} \geq 1 \\ s_{1}+\ldots+s_{m}=i+j-m}} P_{i j}\left(s_{1}, \ldots, s_{m}\right) \partial \partial_{s_{1}}^{\hbar} F \ldots \partial \partial_{s_{m}}^{\hbar} F
$$

for the function $F(x ; \boldsymbol{t})$.

Proof. Since $\Delta(z)=\sum_{k \geq 1} \frac{z^{-k}}{k} \partial_{k}^{\hbar}$, the Hirota equation (26) yields

$$
\begin{gathered}
e^{\Delta\left(z_{1}\right) \Delta\left(z_{2}\right) F}=1+z_{1}^{-1} z_{2}^{-1} \sum_{j=1}^{\infty} \frac{1}{j} \frac{\left(z_{1}^{-j}-z_{2}^{-j}\right)}{\left(z_{1}^{-1}-z_{2}^{-1}\right)} \partial \partial_{j}^{\hbar} F \\
=1+z_{1}^{-1} z_{2}^{-1} \sum_{j=1}^{\infty} \frac{1}{j}\left(\sum_{\substack{s+v=j-1 \\
s, v \geq 0}} z_{1}^{-s} z_{2}^{-v}\right) \partial \partial_{j}^{\hbar} F=1+\sum_{j=1}^{\infty} \frac{1}{j}\left(\sum_{\substack{s+v=j+1 \\
s, v \geq 1}} z_{1}^{-s} z_{2}^{-v}\right) \partial \partial_{j}^{\hbar} F .
\end{gathered}
$$

Therefore,

$$
\Delta\left(z_{1}\right) \Delta\left(z_{2}\right) F=\sum_{m=1}^{\infty} \frac{(-1)^{m+1}}{m}\left(\sum_{n=1}^{\infty}\left(\sum_{\substack{s+v=n+1 \\ s, v \geq 1}} z_{1}^{-s} z_{2}^{-v}\right) \frac{1}{n} \partial \partial_{n}^{\hbar} F\right)^{m}
$$

\footnotetext{
${ }^{1}$ This means that the sequence of operators $h_{0}, h_{1}, h_{2}, \ldots$ is a Hasse-Schmidt derivation, see, e.g., [11.
} 


$$
=\sum_{j=1}^{\infty} \frac{(-1)^{m+1}}{m} \sum_{i, j \geq 1} z_{1}^{-i} z_{2}^{-j} \sum_{\substack{i_{1}+\ldots+i_{m}=i \\ j_{1}+\ldots+j_{m}=j \\ i_{k}, j_{k} \geq 1}} \frac{\partial \partial_{i_{1}+j_{1}-1}^{\hbar} F}{i_{1}+j_{1}-1} \cdots \frac{\partial \partial_{i_{m}+j_{m}-1}^{\hbar} F}{i_{m}+j_{m}-1}
$$

that is

$$
\partial_{i}^{\hbar} \partial_{j}^{\hbar} F=\sum_{m=1}^{\infty} \sum_{k_{1}+\ldots+k_{m}=i+j-m} \frac{(-1)^{m+1} i j}{m k_{1} \ldots k_{m}} \tilde{P}_{i j}\left(k_{1}, \ldots, k_{m}\right) \partial \partial_{k_{1}}^{\hbar} F \ldots \partial \partial_{k_{m}}^{\hbar} F .
$$

This proves that (26) implies (35). Performing the calculation in reverse order, we find that (35) implies (26) .

In particular, we have $\partial_{1} \partial_{j}^{\hbar} F=\partial \partial_{j}^{\hbar} F$.

Let $K_{l}\left(l^{1}, \ldots, l^{r}\right)$ be the number of partitions of a set of $l$ elements into ordered groups of $l^{1}, \ldots, l^{r}$ elements. Define constants $P_{i_{1} \ldots i_{k}}^{\hbar}\left(\begin{array}{c}s_{1} \ldots s_{m} \\ l_{1} \ldots l_{m}\end{array}\right)$ by the following recurrence relations:

1) $P_{i_{1}, i_{2}}^{\hbar}\left(\begin{array}{c}s_{1} \ldots s_{m} \\ 1 \ldots 1\end{array}\right)=P_{i_{1} i_{2}}\left(s_{1}, \ldots, s_{m}\right)$ and $P_{i_{1}, i_{2}}^{\hbar}\left(\begin{array}{c}s_{1} \ldots s_{m} \\ l_{1} \ldots l_{m}\end{array}\right)=0$, if $\prod_{j=1}^{m} l_{j}>1$.

2) $P_{i_{1} \ldots i_{r}}^{\hbar}\left(\begin{array}{l}x_{1} \ldots x_{v} \\ y_{1} \ldots y_{v}\end{array}\right)=\sum P_{i_{1} \ldots i_{r-1}}^{\hbar}\left(s_{1} \ldots s_{m} l_{1} \ldots l_{m}\right) \frac{\hbar^{\nu\left(k_{1}, \ldots, k_{m}\right)-1} i_{r}}{\left[k_{1} \ldots k_{m}\right]}$ $\times K_{l_{1}}\left(l_{1}^{1}, \ldots, l_{1}^{n_{1}}\right) P_{s_{1} k_{1}}^{\hbar}\left(s_{1}^{1} \ldots s_{n_{1}}^{1}\right) \ldots K_{l_{m}}\left(l_{m}^{1}, \ldots, l_{m}^{n_{m}}\right) P_{s_{m} k_{m}}^{\hbar}\left(s_{1}^{m} \ldots s_{n_{m}}^{m}\right)$,

where the summation is carried over all sets of integer numbers $m>0, s_{i}>0, s_{j}^{i}>0$, $k_{i} \geq 0, l_{i}>0, l_{j}^{i} \geq 0$ such that

$$
\begin{gathered}
\left(x_{1} \ldots x_{v}\right)=\left(s_{1}^{1}, \ldots s_{n_{1}}^{1}, s_{1}^{2}, \ldots, s_{n_{2}}^{2}, \ldots, s_{1}^{m}, \ldots, s_{n_{m}}^{m}\right), \quad s_{i}=\sum_{j=1}^{n_{i}} s_{j}^{i} \\
\left(y_{1} \ldots y_{v}\right)=\left(l_{1}^{1}+1, \ldots, l_{n_{1}}^{1}+1, l_{1}^{2}+1, \ldots l_{n_{2}}^{2}+1, \ldots, l_{1}^{m}+1, \ldots, l_{n_{m}}^{m}+1\right), \quad l_{i}=\sum_{j=1}^{n_{i}} l_{j}^{i} \\
\sum_{i=1}^{m}\left(s_{i}+l_{i}\right)=\sum_{j=1}^{r-1} i_{j}, \quad \sum_{i=1}^{m} k_{i}=i_{r}, \quad \sum_{i=1}^{n_{j}} s_{i}^{j}=k_{j}+s_{j} .
\end{gathered}
$$

Lemma 3.2 and Lemma 3.1 imply

Theorem 3.3 The $\hbar$-KP hierarchy (26) is equivalent to the system of equations

$$
\partial_{i_{1}}^{\hbar} \partial_{i_{2}}^{\hbar} \ldots \partial_{i_{r}}^{\hbar} F=\sum_{m \geq 1} \sum_{\begin{array}{c}
s_{1}+l_{1}+\ldots+s_{m}+l_{m} \\
=i_{1}+\ldots+i_{r} \\
1 \leq s_{i} ; 1 \leq l_{i} \leq r-1
\end{array}} P_{i_{1} \ldots i_{r}}^{\hbar}\left(\begin{array}{c}
s_{1} \ldots s_{m} \\
l_{1} \ldots l_{m}
\end{array}\right) \partial^{l_{1}} \partial_{s_{1}}^{\hbar} F \ldots \partial^{l_{m}} \partial_{s_{m}}^{\hbar} F
$$

(here $r \geq 2$ ). 
Proof. We use induction in $r$. At $r=2$ Lemma 3.2 gives

$$
\partial_{i_{1}}^{\hbar} \partial_{i_{2}}^{\hbar} F=\sum_{m \geq 1} \sum_{\substack{s_{1}+\ldots+s_{m}+1 \\
=i_{1}+i_{2} \\
1 \leq s_{i}}} P_{i_{1} i_{2}}^{\hbar}\left(\begin{array}{c}
s_{1} \ldots s_{m} \\
1 \ldots 1
\end{array}\right) \partial \partial_{s_{1}}^{\hbar} F \ldots \partial \partial_{s_{m}}^{\hbar} F
$$

Then we calculate $\partial_{i_{1}}^{\hbar} \partial_{i_{2}}^{\hbar} \ldots \partial_{i_{r}}^{\hbar} F=\partial_{i_{r}}^{\hbar}\left(\partial_{i_{1}}^{\hbar} \partial_{i_{2}}^{\hbar} \ldots \partial_{i_{r-1}}^{\hbar} F\right)$ assuming that $\partial_{i_{1}}^{\hbar} \partial_{i_{2}}^{\hbar} \ldots \partial_{i_{r-1}}^{\hbar} F$ is given by the assertion of the theorem. We have, using the generalized Leibniz rule (34):

$$
\begin{aligned}
& \partial_{i_{1}}^{\hbar} \partial_{i_{2}}^{\hbar} \ldots \partial_{i_{r}}^{\hbar} F=\partial_{i_{r}}^{\hbar}\left(\partial_{i_{1}}^{\hbar} \partial_{i_{2}}^{\hbar} \ldots \partial_{i_{r-1}}^{\hbar} F\right) \\
& =\sum_{m \geq 1} \sum_{\begin{array}{c}
s_{1}+l_{1}+\ldots+s_{m}+l_{m} \\
i_{1}+\ldots+i_{r}-1 \\
1 \leq s_{i} ; 1 \leq l_{i} \leq r-2
\end{array}} P_{i_{1} \ldots i_{r-1}}^{\hbar}\left(\begin{array}{c}
s_{1} \ldots s_{m} \\
l_{1} \ldots l_{m}
\end{array}\right) \partial_{i_{r}}^{\hbar}\left(\partial^{l_{1}} \partial_{s_{1}}^{\hbar} F \ldots \partial^{l_{m}} \partial_{s_{m}}^{\hbar} F\right) \\
& =\sum_{m \geq 1} \sum_{\substack{s_{1}+l_{1}+\ldots+s_{m}+l_{m} \\
=i_{1}+\ldots+i_{r}-1 \\
1 \leq s_{i} ; 1 \leq l_{i} \leq r-2}} P_{i_{1} \ldots i_{r-1}}^{\hbar}\left(\begin{array}{c}
s_{1} \ldots s_{m} \\
l_{1} \ldots l_{m}
\end{array}\right) \sum_{\substack{k_{1}, \ldots, k_{m} \geq 0 \\
k_{1}+\ldots+k_{m}=i_{r}}} \frac{\hbar^{\nu\left(k_{1}, \ldots, k_{m}\right)-1} i_{r}}{\left[k_{1} \ldots k_{m}\right]} \\
& \partial^{l_{1}} \partial_{s_{1}}^{\hbar} \partial_{k_{1}}^{\hbar} F \ldots \partial^{l_{m}} \partial_{s_{m}}^{\hbar} \partial_{k_{m}}^{\hbar} F \\
& =\sum_{m \geq 1} \sum_{\substack{s_{1}+l_{1}+\ldots+s_{m}+l_{m} \\
=i_{1}+\ldots,+i_{r-1} \\
1 \leq s_{i} ; 1 \leq l_{i} \leq r-2}} P_{i_{1} \ldots i_{r-1}}^{\hbar}\left(\begin{array}{c}
s_{1} \ldots s_{m} \\
l_{1} \ldots l_{m}
\end{array}\right) \sum_{\substack{k_{1}, \ldots, k_{m} \geq 0 \\
k_{1}+\ldots+k_{m}=i_{r}}} \frac{\hbar^{\nu\left(k_{1}, \ldots, k_{m}\right)-1} i_{r}}{\left[k_{1} \ldots k_{m}\right]} \\
& \partial^{l_{1}}\left(\sum_{n_{1} \geq 1} \sum_{\substack{s_{1}, \ldots, s_{n_{1}} \geq 1 \\
s_{1}^{1}+\ldots+s_{n_{1}}^{1}+n_{1}=k_{1}+s_{1}}} P_{s_{1} k_{1}}^{\hbar}\left(s_{1}^{1} \ldots s_{n_{1}}^{1}\right) \partial \partial_{s_{1}^{1}}^{\hbar} F \ldots \partial \partial_{s_{n_{1}}^{1}}^{\hbar} F\right) \ldots \\
& \partial^{l_{m}}\left(\sum_{n_{m} \geq 1} \sum_{\substack{1 \\
s_{1}^{m}, \ldots, s_{n m}^{m} \geq 1 \\
s_{1}^{m}+\ldots+s_{n_{m}}^{m}+n_{m}=k_{m}+s_{m}}} P_{s_{m} k_{m}}^{\hbar}\left(s_{1}^{m} \ldots s_{n_{m}}^{m}\right) \partial \partial_{s_{1}^{m}}^{\hbar} F \ldots \partial \partial_{s_{n_{m}}^{m}}^{\hbar} F\right)
\end{aligned}
$$

which is equivalent to (36).

\section{Formal solution for the $F$-function}

\subsection{The Cauchy-like data}

Similarly to Definition 2.1, we can introduce the Cauchy-like data for the $\hbar$-KP hierarchy for $F: F(x ; \mathbf{0})$ and $\partial_{k}^{\hbar} F(x ; \mathbf{0}):=\left.\partial_{k}^{\hbar} F(x ; \mathbf{t})\right|_{\mathbf{t}=0}$. Let us examine how the Cauchy-like data for $\tau$ and $F$ are connected. First of all, we have

$$
\tau(x ; \mathbf{0})=c_{0}(x)=e^{F(x ; \mathbf{0}) / \hbar^{2}} .
$$


Next, we use the obvious identity $\hbar \frac{\Delta(z) \tau}{\tau}=e^{\frac{1}{\hbar} \Delta(z) F}-1$ which immediately follows from the definition of $\Delta(z) 2$. Expanding it in powers of $z$ and comparing the coefficients, we get:

$$
\left.\frac{\partial_{k}^{\hbar} \tau(x ; \mathbf{t})}{\tau(x ; \mathbf{0})}\right|_{\mathbf{t}=0}=\frac{k}{\hbar} h_{k}(\mathbf{y}), \quad y_{l}=\left.\frac{1}{\hbar l} \partial_{l}^{\hbar} F(x ; \mathbf{t})\right|_{\mathbf{t}=0},
$$

or, in terms of the coefficients $c_{k}(x)$ introduced in Theorem 2.1 ,

$$
\frac{c_{k}(x)}{c_{0}(x)}=h_{k}(\mathbf{y}), \quad k=1,2, \ldots
$$

Therefore, the Cauchy-like data for $\tau$ are unambiguously determined by the ones for $F$. Theorem 2.1 implies the following

Corollary 4.1 Let $\hbar \neq 0$. Then for any Cauchy-like data $F(x ; \mathbf{0}), \partial_{k}^{\hbar} F(x ; \mathbf{0}), k \geq 1$, there exists a formal solution $F(x ; \mathbf{t})$ to the $\hbar$-KP hierarchy.

\subsection{Dual bases $h_{\lambda}$ and $m_{\lambda}$ and the formal solution}

Theorem 3.3 gives $h_{\lambda}(\hbar \tilde{\partial}) F(x ; \mathbf{0})$ in terms of the Cauchy-like data. Therefore, for the construction of the Taylor series we need the dual bases $h_{\lambda}, m_{\mu}$. The functions $m_{\mu}(\mathbf{t})$ will be used in the expansion of the $F$-function instead of monomials $t_{\lambda}$, according to (10).

It is not difficult to incorporate the parameter $\hbar$ into the formulas. For instance, re-scaling $\mathbf{t} \rightarrow \mathbf{t} / \hbar$ in the generalized Taylor expansion (10) with respect to a dual pair $\left\langle u_{\lambda}, v_{\mu}\right\rangle=\delta_{\lambda \mu}$ and redefining the function $g$ as $g(\mathbf{t} / \hbar)=G(\mathbf{t})$, we can rewrite it in the form

$$
G(\mathbf{t})=\left.\sum_{\lambda} u_{\lambda}(\hbar \tilde{\partial}) G\left(\mathbf{t}^{\prime}\right)\right|_{\mathbf{t}^{\prime}=0} \cdot v_{\lambda}(\mathbf{t} / \hbar)
$$

Here, $G(\mathbf{t})$ is an arbitrary series in the $t_{j}$ 's with $\hbar$-dependent coefficients. Applying this formula for the dual pair $h_{\lambda}, m_{\lambda}$ and the function $F(x ; \mathbf{t})$, we can write:

$$
F(x ; \mathbf{t})=\left.\sum_{\lambda} h_{\lambda}(\hbar \tilde{\partial}) F\left(x ; \mathbf{t}^{\prime}\right)\right|_{\mathbf{t}^{\prime}=0} \cdot m_{\lambda}(\mathbf{t} / \hbar) .
$$

As the $\hbar$-deformation of the monomial basis $t_{\lambda}$ we introduce the polynomials

$$
t_{\lambda}^{\hbar}:=\frac{\sigma(\lambda)}{\rho(\lambda)} \hbar^{\ell(\lambda)} m_{\lambda}(\mathbf{t} / \hbar)
$$

The first few are (see (요) ):

$$
\begin{aligned}
& t_{(1)}^{\hbar}=t_{1} \\
& t_{(2)}^{\hbar}=t_{2}, \quad t_{\left(1^{2}\right)}^{\hbar}=t_{1}^{2}-2 \hbar t_{2} \\
& t_{(3)}^{\hbar}=t_{3}, \quad t_{(21)}^{\hbar}=t_{2} t_{1}-\frac{3}{2} \hbar t_{3}, \quad t_{\left(1^{3}\right)}^{\hbar}=t_{1}^{3}-6 \hbar t_{2} t_{1}+6 \hbar^{2} t_{3} .
\end{aligned}
$$

\footnotetext{
${ }^{2}$ It is valid for any functions $\tau, F$ related by $F=\hbar^{2} \log \tau$ (not necessarily KP solutions).
} 
Clearly, $t_{\lambda}^{\hbar=0}=t_{\lambda}$. In these terms, the Taylor series for the function $F$ can be written as follows:

$$
F(x ; \mathbf{t})=\left.\sum_{\lambda} \partial_{\lambda}^{\hbar} F\left(x ; \mathbf{t}^{\prime}\right)\right|_{\mathbf{t}^{\prime}=0} \frac{t_{\lambda}^{\hbar}}{\sigma(\lambda)},
$$

where $\partial_{\lambda}^{\hbar}:=\partial_{\lambda_{1}}^{\hbar} \partial_{\lambda_{2}}^{\hbar} \ldots \partial_{\lambda_{\ell}}^{\hbar}$. All solutions of system (35) of this form are called formal solutions. An important corollary of Theorem 3.3 is

Corollary 4.2 Any formal solution $F(x ; \boldsymbol{t})$ of (26) is uniquely defined by the Cauchy-like data $F(x, \mathbf{0})$ and $\left\{\partial_{k}^{\hbar} F(x ; \mathbf{0}) \mid k=1,2, \ldots\right\}$.

\section{$4.3 t_{\lambda}^{\hbar}$ in terms of $t_{\lambda}$}

Following [10], consider the transition matrix $L_{\lambda \mu}$ from power sums to the monomial symmetric functions:

$$
p_{\lambda}(\mathbf{t})=\sum_{\mu} L_{\lambda \mu} m_{\mu}(\mathbf{t})
$$

It has the following combinatorial description. Let $\lambda$ be a partition of length $\ell=\ell(\lambda)$, and $f$ be any mapping from the set $\{1,2, \ldots, \ell\}$ to the set of positive integers. Consider the infinite sequence $\left\{f_{1}^{(\lambda)}, f_{2}^{(\lambda)}, \ldots\right\}$ whose $i$ th component is

$$
f_{i}^{(\lambda)}=\sum_{j: f(j)=i} \lambda_{j} \quad \text { for each } i \geq 1 .
$$

Theorem 4.1 ([10]) $L_{\lambda \mu}$ is equal to the number of mappings $f$ such that $f_{i}^{(\lambda)}=\mu_{i}$ for each $i \geq 1$.

It follows from the theorem that $L_{\lambda \mu}$ are non-negative integer numbers. Let us regard the partitions of $n=|\lambda|$ as arranged in the reverse lexicographical order. It follows from the theorem that $L_{\lambda \mu}=0$ unless $\mu$ precedes $\lambda$. In fact a stronger property holds: $L_{\lambda \mu}=0$ unless $\mu \geq \lambda$, where the partial ordering $\geq$ is defined in (3). This means that the matrix $L_{\lambda \mu}$ is strictly lower triangular:

$$
p_{\lambda}(\mathbf{t})=\sum_{\mu \geq \lambda} L_{\lambda \mu} m_{\mu}(\mathbf{t}), \quad m_{\lambda}(\mathbf{t})=\sum_{\mu \geq \lambda}\left(L^{-1}\right)_{\lambda \mu} p_{\mu}(\mathbf{t})
$$

(in particular, $L_{\lambda \mu}=\left(L^{-1}\right)_{\lambda \mu}=0$ if $\lambda$ and $\mu$ are incomparable with respect to the partial ordering $\geq$ ). We can also rewrite (46) as

$$
t_{\lambda}^{\hbar}=\frac{\sigma(\lambda)}{\rho(\lambda)} \sum_{\mu \geq \lambda}\left(L^{-1}\right)_{\lambda \mu} \rho(\mu) \hbar^{\ell(\lambda)-\ell(\mu)} t_{\mu} .
$$

Let us stress that the sums in (46), (47) are finite: it is implied that $|\mu|=|\lambda|$. 


\subsection{Algebra of $\hbar$-differential operators}

Let $F$ be a solution of the $\hbar$-KP hierarchy. It follows from Theorem 3.3 that $\left\{\partial_{i}^{\hbar} \mid i=\right.$ $1,2, \ldots\}$ act on the algebra generated by $\left\{\partial_{1}^{l} \partial_{s}^{\hbar} F\left(x ; t_{1}, 0,0, \ldots\right) \mid l, s=1,2, \ldots\right\}$. In this subsection we prove that any family of functions of one variable generates a similar commutative algebra of differential operators. Later we use this algebra for constructing all formal solutions.

Consider an arbitrary set $\mathbf{f}=\left\{f_{1}, f_{2}, \ldots\right\}$ of real or complex formal functions of the variable $x$. Let $A_{\mathbf{f}}$ be the algebra generated by $\left\{f_{i}\right\}$ and their derivatives $\partial^{n} f_{i}=\frac{\partial^{n} f_{i}}{\partial x^{n}}$.

Consider a family of linear operators $L_{i}^{\hbar}=L_{i}^{\hbar}[\mathbf{f}]: A_{\mathbf{f}} \rightarrow A_{\mathbf{f}}$, which are uniquely defined by the following properties:

$$
\begin{aligned}
& \text { - } L_{i}^{\hbar} \partial=\partial L_{i}^{\hbar} \\
& \text { - } L_{i}^{\hbar}\left(f_{j}\right)=\sum_{m \geq 1} \sum_{\substack{s_{1}, \ldots, s_{m}>1 \\
s_{1}+\ldots+s_{m}=i+j}} P_{i j}\left(s_{1}-1, \ldots, s_{m}-1\right) \partial f_{s_{1}-1} \ldots \partial f_{s_{m}-1}
\end{aligned}
$$

- (the generalized Leibniz rule) for any formal functions $g_{1}, \ldots, g_{n}$, of $t$ and $\hbar$ it holds

$$
L_{k}^{\hbar}\left(g_{1} \ldots g_{n}\right)=\sum_{\substack{k_{1}, \ldots, k_{n} \geq 0 \\ k_{1}+\ldots, k_{n}=k}} \frac{\hbar^{\nu\left(k_{1}, \ldots, k_{n}\right)-1} k}{\left[k_{1} \ldots k_{n}\right]} L_{k_{1}}^{\hbar} g_{1} L_{k_{2}}^{\hbar} g_{2} \ldots L_{k_{n}}^{\hbar} g_{n}
$$

In particular, $L_{1}^{\hbar}\left(f_{j}\right)=\sum_{m \geq 1} \sum_{\substack{s_{1}, \ldots, s_{m}>1 \\ s_{1}+\ldots+s_{m}=1+j}} P_{1 j}\left(s_{1}-1, \ldots, s_{m}-1\right) \partial f_{s_{1}-1} \ldots \partial f_{s_{m}-1}=\partial f_{j}$ and $L_{j}^{\hbar}\left(f_{i}\right)=L_{i}^{\hbar}\left(f_{j}\right)$ because $P_{j i}\left(s_{1}, \ldots s_{m}\right)=P_{i j}\left(s_{1}, \ldots s_{m}\right)$.

The lemma below follows from Lemma 3.2. (This is a motivation for introducing the operators $L_{i}^{\hbar}$.)

Lemma 4.1 Let $F$ be the solution to the $\hbar$-KP hierarchy corresponding to the Cauchylike data $F(x, \mathbf{0})$ and $\left\{\partial_{k}^{\hbar} F(x, \mathbf{0})=f_{k}(x) \mid k=1,2, \ldots\right\}$. Then

$$
L_{i}^{\hbar}\left(f_{j}\right)(x)=\partial_{i}^{\hbar} \partial_{j}^{\hbar} F(x, \mathbf{0}) .
$$

Theorem 4.2 The operators $L_{i}^{\hbar}$ commute with each other.

Proof. First assume that $\hbar \neq 0$. Then it follows from Corollary 4.1 that there is a unique solution $F$ to the $\hbar$-KP hierarchy with the Cauchy-like data f. Hence

$$
L_{k}^{\hbar} L_{i}^{\hbar}\left(f_{j}\right)=\partial_{k}^{\hbar} \partial_{i}^{\hbar} \partial_{j}^{\hbar} F(x ; \mathbf{t})=L_{i}^{\hbar} L_{k}^{\hbar}\left(f_{j}\right)
$$

The operators $L_{i}^{\hbar}$ are presented in the form of formal series $L_{i}^{\hbar}=\sum_{j=0}^{\infty} \hbar^{j} H_{i}^{j}$, where the linear operators $H_{i}^{j}=H_{i}^{j}[\mathbf{f}]: A_{\mathbf{f}} \rightarrow A_{\mathbf{f}}$ do not depend on $\hbar$ and $H_{i}^{0}=L_{i}^{0}$. The equalities $L_{k}^{\hbar} L_{i}^{\hbar}\left(f_{j}\right)=L_{i}^{\hbar} L_{k}^{\hbar}\left(f_{j}\right)$ for all non-zero $\hbar$ give $H_{k}^{0} H_{i}^{0}\left(f_{j}\right)=H_{i}^{0} H_{k}^{0}\left(f_{j}\right)$. 
Corollary 4.3 For any $i_{1}, \ldots, i_{r}$ and $\sigma \in S_{r}$ the relations

$$
L_{i_{1}}^{\hbar} \ldots L_{i_{r-1}}^{\hbar}\left(f_{i_{r}}\right)=L_{i_{\sigma(1)}}^{\hbar} \ldots L_{i_{\sigma(r-1)}}^{\hbar}\left(f_{i_{\sigma(r)}}\right)
$$

and

$$
L_{i_{1}}^{\hbar} \ldots L_{i_{r-1}}^{\hbar}\left(f_{i_{r}}\right)=\sum_{m \geq 1} \sum_{\substack{s_{1}+l_{1}+\ldots+s_{m}+l_{m}=\\
1 \leq i_{i} ; 1 \leq l_{i} \leq i_{r}-1}} P_{i_{1} \ldots i_{r}}^{\hbar}\left(\begin{array}{c}
s_{1} \ldots s_{m} \\
l_{1} \ldots l_{m}
\end{array}\right) \partial^{l_{1}} f_{s_{1}} \ldots \partial^{l_{m}} f_{s_{m}}
$$

are fulfilled.

Proof. The first relation directly follows from Theorem 4.2. The second one follows from Lemma 4.1 and Theorem 3.3.

\subsection{Construction of formal solutions}

Given a Young diagram $\lambda=\left[\lambda_{1}, \ldots, \lambda_{\ell}\right]$, we put $P_{\lambda}^{\hbar}\left(\begin{array}{c}s_{1} \ldots s_{m} \\ l_{1} \ldots l_{m}\end{array}\right)=P_{\lambda_{1} \ldots \lambda_{r}}^{\hbar}\left(\begin{array}{c}s_{1} \ldots s_{m} \\ l_{1} \ldots l_{m}\end{array}\right)$.

Theorem 4.3 For any $\hbar$ and any family of smooth or formal functions

$$
\boldsymbol{f}=\left\{f_{0}(x), f_{1}(x), f_{2}(x), \ldots\right\}
$$

there exists a unique solution $F(x ; \mathbf{t})$ of the $\hbar$-KP hierarchy such that $F(x ; \mathbf{0})=f_{0}(x)$ and $\left.\partial_{k}^{\hbar} F\left(x ; t_{1}, t_{2}, \ldots\right)\right|_{\mathbf{t}=0}=f_{k}(x)$. This solution has the form

$$
F(x ; \mathbf{t})=f_{0}(x)+\sum_{|\lambda| \geq 1} \frac{f_{\lambda}^{\hbar}(x)}{\sigma(\lambda)} t_{\lambda}^{\hbar}
$$

where $f_{(k)}^{\hbar}(x)=f_{k}(x)$ and

$$
f_{\lambda}^{\hbar}(x)=\sum_{m \geq 1} \sum_{\substack{s_{1}+l_{1}+\ldots+s_{m}+l_{m}=|\lambda| \\
1 \leq s_{i} ; 1 \leq l_{i} \leq \ell(\lambda)-1}} P_{\lambda}^{\hbar}\left(\begin{array}{c}
s_{1} \ldots s_{m} \\
l_{1} \ldots l_{m}
\end{array}\right) \partial^{l_{1}} f_{s_{1}}(x) \ldots \partial^{l_{m}} f_{s_{m}}(x)
$$

for $\ell(\lambda)>1$.

Proof. Let us consider the operators $L_{i}^{\hbar}$ generated by the family $\left\{f_{i} \mid i=0,1,2, \ldots\right\}$. Given a Young diagram $\lambda=\left[\lambda_{1}, \ldots, \lambda_{r}\right]$, denote by $[i, j, \lambda]$ the Young diagram with the rows $\left\{i, j, \lambda_{1}, \ldots, \lambda_{r}\right\}$ (ordered according to their length). Then, according to Corollary 4.3 ,

$$
\partial_{i}^{\hbar} \partial_{j}^{\hbar} F=\sum_{\lambda} \frac{f_{[i, j, \lambda]}(x)}{\sigma([i, j, \lambda])} t_{\lambda}^{\hbar}=\sum_{\lambda} \frac{L_{i}^{\hbar} L_{j}^{\hbar} f_{\lambda}^{\hbar}(x)}{\sigma(\lambda)} t_{\lambda}^{\hbar}=L_{i}^{\hbar} L_{j}^{\hbar} \sum_{\lambda} \frac{f_{\lambda}^{\hbar}(x)}{\sigma(\lambda)} t_{\lambda}^{\hbar},
$$

for $i, j \geq 1$. Hence,

$$
\left.\partial_{i}^{\hbar} \partial_{j}^{\hbar} F\left(x ; t_{1}, t_{2}, \ldots\right)\right|_{\mathbf{t}=0}=L_{i}^{\hbar} L_{j}^{\hbar} F(x ; \mathbf{0})
$$


and

$$
\partial_{i}^{\hbar} \partial_{j}^{\hbar} F=\sum_{m \geq 1} \sum_{\substack{s_{1}, \ldots, s_{m}>1 \\ s_{1}+\ldots+s_{m}=i+j}} P_{i j}\left(s_{1}-1, \ldots, s_{m}-1\right) \partial \partial_{s_{1}-1}^{\hbar} F \ldots \partial \partial_{s_{m}-1}^{\hbar} F^{\hbar}
$$

Therefore, according to Lemma 3.2, the function $F$ is a formal solution of the $\hbar$-KP hierarchy. Moreover, according to Corollary 4.2, the solution is uniquely determined by the functions $f_{k}(x)$.

Corollary 4.4 The Cauchy data are connected with the Cauchy-like data by

$$
\left.\partial_{k} F(x ; \mathbf{t})\right|_{\mathbf{t}=0}=k \sum_{|\lambda|=k} \frac{\kappa_{\lambda}}{\rho(\lambda)} f_{\lambda}^{\hbar}(x) \hbar^{\ell(\lambda)-1}
$$

where $\kappa_{\lambda}=\left(L^{-1}\right)_{\lambda(k)}$. For any family formal functions $\boldsymbol{f}=\left\{f_{0}(x), f_{1}(x), f_{2}(x), \ldots\right\}$ there exists a single solution of $\hbar-K P$ hierarchy $F(x ; \mathbf{t})$ with Cauchy data $\boldsymbol{f}$.

Proof. The first statement immediately follows from (47) and (49). According to Theorem 4.3, the second statement follows from an inversion of (51). This inversion is proved by induction in $k$, according to

$$
f_{k}^{\hbar}(x)=\left.\partial_{k} F(x ; \mathbf{t})\right|_{\mathbf{t}=0}-k \sum_{\substack{|\lambda|=k \\ \ell(\lambda)>1}} \frac{\kappa_{\lambda}}{\rho(\lambda)} f_{\lambda}^{\hbar}(x) \hbar^{\ell(\lambda)-1}
$$

\section{Acknowledgments}

The work of S.N. was supported in part by RFBR grant 15-52-50041, and by grant Nsh-5138.2014.1 for support of scientific schools. The work of A.Z. was supported in part by RFBR grants 14-02-00627, 15-52-50041-YaF, 14-01-90405-Ukr and by grant Nsh1500.2014.2 for support of scientific schools. The financial support from the Government of the Russian Federation within the framework of the implementation of the 5-100 Programme Roadmap of the National Research University Higher School of Economics is acknowledged.

\section{Appendix A: Proof of Proposition 2.2}

Here we give some details of the proof that the tau-function of the $\hbar$-KP hierarchy satisfies (14) for any $m \geq 2$ :

$$
\prod_{1 \leq i<j \leq m}\left(z_{j}-z_{i}\right) \cdot \tau^{\left[z_{1}, \ldots, z_{m}\right]} \tau^{m-1}=\operatorname{det}_{1 \leq j, k \leq m}\left(\left(z_{j}-\hbar \partial_{1}\right)^{k-1} \tau^{\left[z_{j}\right]}\right)
$$


(Proposition 2.2). At $m=2$ equation (A1) reads

$$
\left(z_{2}-z_{1}\right) \tau^{\left[z_{1}, z_{2}\right]} \tau=\left|\begin{array}{ll}
\tau^{\left[z_{1}\right]} & \left(z_{1}-\hbar \partial_{1}\right) \tau^{\left[z_{1}\right]} \\
\tau^{\left[z_{2}\right]} & \left(z_{2}-\hbar \partial_{1}\right) \tau^{\left[z_{2}\right]}
\end{array}\right|
$$

which is the original equation for the tau-function (13). The rest of the proof is induction in $m$.

Suppose (A1) holds for any number of points $z_{i}$ from 2 to $m-1$. In what follows it is convenient to use the short-hand notation

$$
\Delta_{1 \ldots m}=\prod_{a>b}^{m}\left(z_{a}-z_{b}\right), \quad \tau_{1 \ldots m}=\tau\left(\mathbf{t}+\hbar\left[z_{1}^{-1}\right]+\ldots+\hbar\left[z_{m}^{-1}\right]\right) .
$$

After the shift $\mathbf{t} \rightarrow \mathbf{t}+\sum_{i=3}^{m}\left[z_{i}^{-1}\right]$ equation (A2) acquires the form

$$
\Delta_{12} \tau_{123 \ldots m} \tau_{3 \ldots m}=\left|\begin{array}{ll}
\tau_{13 \ldots m} & \left(z_{1}-\hbar \partial_{1}\right) \tau_{13 \ldots m} \\
\tau_{23 \ldots m} & \left(z_{2}-\hbar \partial_{1}\right) \tau_{23 \ldots m}
\end{array}\right| .
$$

Let $N_{i j}$ be the matrix $N_{i j}=\left(z_{i}-\hbar \partial_{1}\right)^{j-1} \tau^{\left[z_{i}\right]}, 1 \leq i, j \leq m$, and $\tilde{N}_{i j}$ be the matrix whose first $m-1$ columns are the same as for $N_{i j}$ but the last column is different: $\tilde{N}_{i m}=N_{i m+1}$. One can check 3 that

$$
\hbar \partial_{1} \operatorname{det} N_{i j}=\left(\sum_{l=1}^{m} z_{l}\right) \operatorname{det} N_{i j}-\operatorname{det} \tilde{N}_{i j}
$$

By $N_{i j}[a, b]$ we denote the matrix $N_{i j}$ with removed row $a$ and column $b$, and by $N_{i j}\left[\begin{array}{l}a, b \\ c, d\end{array}\right]$ we denote the matrix $N_{i j}$ with removed rows $a, c$ and column $b, d$. By the assumption of the induction we have:

$$
\tau_{3 \ldots m}=\frac{\operatorname{det} N_{i j}\left[\begin{array}{cc}
1, & m-1 \\
2, & m
\end{array}\right]}{\Delta_{3 \ldots m} \tau^{m-3}}, \quad \tau_{13 \ldots m}=\frac{\operatorname{det} N_{i j}[2, m]}{\Delta_{13 \ldots m} \tau^{m-2}}, \quad \tau_{23 \ldots m}=\frac{\operatorname{det} N_{i j}[1, m]}{\Delta_{23 \ldots m} \tau^{m-2}} .
$$

Substituting this into (A3), we get, after using (A4):

$$
\Delta_{12} \tau_{1 \ldots m} \cdot \frac{\operatorname{det} N\left[\begin{array}{cc}
1, & m-1 \\
2, & m
\end{array}\right]}{\Delta_{3 \ldots m} \tau^{m-3}}=\frac{\left|\begin{array}{ll}
\operatorname{det} N[2, m] & \operatorname{det} N[2, m-1] \\
\operatorname{det} N[1, m] & \operatorname{det} N[1, m-1]
\end{array}\right|}{\Delta_{13 \ldots m} \Delta_{23 \ldots m} \tau^{2 m-4}}
$$

Since $\Delta_{12} \Delta_{13 \ldots m} \Delta_{23 \ldots m}=\Delta_{3 \ldots m} \Delta_{1 \ldots m}$, equation (A1) for $m$ points, $\tau_{1 \ldots m}=\frac{\operatorname{det} N}{\Delta_{1 \ldots m} \tau^{m-1}}$, follows from the Jacobi identity for minors of the matrix $N$ :

$$
\operatorname{det} N \cdot \operatorname{det} N\left[\begin{array}{cc}
1, & m-1 \\
2, & m
\end{array}\right]=\left|\begin{array}{ll}
\operatorname{det} N[2, m] & \operatorname{det} N[2, m-1] \\
\operatorname{det} N[1, m] & \operatorname{det} N[1, m-1]
\end{array}\right| .
$$

\footnotetext{
${ }^{3}$ The proof is based on the easily verified identity $\sum_{l=1}^{m} \operatorname{det}\left(z_{i}^{\delta_{j l}} A_{i j}\right)=\left(\sum_{l=1}^{m} z_{l}\right) \operatorname{det} A_{i j}$ valid for any
} matrix $A_{i j}$ 


\section{References}

[1] A. Alexandrov, S. Leurent, Z. Tsuboi and A. Zabrodin, The master Toperator for the Gaudin model and the KP hierarchy, Nucl. Phys. B883 (2014) 173-223.

[2] E. Date, M. Jimbo, M. Kashiwara and T. Miwa, Transformation groups for soliton equations, in "Nonlinear integrable systems - classical and quantum", eds. M. Jimbo and T. Miwa, World Scientific, pp. 39-120 (1983).

[3] B. Dubrovin and S. Natanzon, Real Theta-function of the KadontsevPetviashvilli equation, Math. USSR Izvestiya, 32:2 (1989), 269-288.

[4] V. Enolski and J. Harnad, Schur function expansions of KP tau functions associated to algebraic curves, Uspekhi Mat. Nauk 66:4 (2011) 137-178 (Russian Math. Surveys 66:4 (2011) 767-807), arXiv:1012.3152.

[5] M. Jimbo and T. Miwa, Solitons and infinite dimensional Lie algebras, Publ. RIMS, Kyoto Univ. 19 (1983) 943-1001.

[6] I. Krichever, Methods of algebraic geometry in the theory of non-linear equations, Rus. Math. Serv. 32 (6) (1977) 185-213.

[7] I. Krichever, The method of averaging for two dimensional integrable equations, Funct. Anal. Appl. 22 (1989) 200-213.

[8] I. Krichever, The $\tau$-function of the universal Whitham hierarchy, matrix models and topological field theories, Comm. Pure Appl. Math. 47 (1994) 437-475, arXiv:hep-th/9205110.

[9] S. Lando Ramified coverings of two-dimensional sphere and the intersection theory in spaces of meromorphic functions on algebraic curves Rus. Math. Serv. 57 (3) (2002) 463-533.

[10] I. Macdonald, Symmetric functions and Hall polynomials, 2nd ed., Oxford University Press, 1995.

[11] H. Matsumura, Commutative ring theory, volume 8 of "Cambridge Studies in Advanced Mathematics", Cambridge University Press, Cambridge, 1986.

[12] M. Mineev-Weinstein, P. Wiegmann and A. Zabrodin, Integrable structure of interface dynamics, Phys. Rev. Lett. 84 (2000) 5106-5109, arXiv:nlin.SI/0001007.

[13] M. Mineev-Weinstein and A. Zabrodin, Whitham-Toda hierarchy in the Laplacian growth problem, Proceedings of the Workshop NEEDS-99 (Crete, Greece, June 1999), J. Nonlin. Math. Phys. 8 (2001) 212-218.

[14] A. Morozov Integrability and matrix models, Physics Uspekhi 37 (1994) 155. 
[15] S. Natanzon, Towards an effectivization of the Riemann theorem, Ann. Global Anal. Geom. 28 (2005) 233-255.

[16] S. Natanzon, Integrable systems and effectivization of the Riemann theorem about domains of the complex plane., Moscow. Math. J. 3(2) (2003) 541-549.

[17] S. Natanzon, Formulas for $A_{n}$ and $B_{n}$-solutions of WDVV equations, J. Geom. Phys. 39 (2001) 323-336.

[18] S. Natanzon, Witten solution for the Gelfand-Dikii hierarchy, Funk. Anal. i ego Prilozh., 37:1 (2003) 25-37 (English translation: Functional Analysis and Its Applications 37:1 (2003) 2131).

[19] S. Natanzon and A. Zabrodin, Symmetric solutions to dispersionless 2D Toda hierarchy, Hurwitz numbers and conformal dynamics, Int. Math. Res. Notices 2015 (2015) 2082-2110; arXiv:1302.7288.

[20] S. Novikov, S. Manakov, P. Pitaevsky, V. Zakharov, Theory of solitons: the invers scatteling method, Nauka, Moscow, 1980.

[21] M. Sato, Soliton equations as dynamical systems on a infinite dimensional Grassmann manifold, RIMS Kokyuroku 439 (1981) 30-46.

[22] K. Takasaki and T. Takebe, Integrable hierarchies and dispersionless limit, Rev. Math. Phys. 7 (1995) 743-808.

[23] K. Takasaki and T. Takebe, $\hbar$-expansion of KP hierarchy: Recursive construction of solutions, arXiv:0912.4867.

[24] K. Takasaki and T. Takebe, $\hbar$-expansion of KP hierarchy, Theor. Math. Phys. 171 (2012) 683-690.

[25] K. Takasaki and T. Takebe, $\hbar$-expansion of the Toda hierarchy: Recursive construction of solutions, Anal. Math. Phys. 2 (2012) 171-214.

[26] P. Wiegmann and A. Zabrodin, Conformal maps and integrable hierarchies, Commun. Math. Phys. 213 (2000) 523-538.

[27] E. Witten Two-dimensional gravity and intersection theory on moduli spaces, Surveys in differential geometry, 1, International Press, Inc., Boston, MA, 1991, 5243-310.

[28] A. Zabrodin, Growth of fat slits and dispersionless KP hierarchy, J. Phys. A: Math. Theor. 42 (2009) 085206 (23pp), arXiv:0811.4054.

[29] A. Zabrodin, Laplacian growth in a channel and Hurwitz numbers, J. Phys. A: Math. Theor. 46 (2013) 185203 (23pp), arXiv:1212.6729.

[30] A. Zabrodin, The dispersionless limit of the Hirota equations in some problems of complex analysis, Theor. Math. Phys. 129 (2001) 1511-1525 (Teor. Mat. Fiz. 129 (2001) 239-257), arXiv:math.CV/0104169. 